\title{
Archivos de Cardiología de México

\section{Declaración ante los resultados del ensayo clínico Symplicity HTN 3. Grupo de estudio de la hipertensión arterial resistente}

\author{
José Ramón Azpiri-López*, José Luis Assad-Morell, Enrique Ponce de \\ León-Martínez, Rogelio Monreal-Puente, Adrián Dávila-Bortoni, \\ Luis Alberto Vázquez-Díaz, Ramón Javier Treviño-Frutos, Félix Barrera-Oranday, \\ Juan Gustavo del Angel-Soto, José Guadalupe Martínez y Marcelo Arellano-Torres
}

Hospital Christus Muguerza Alta Especialidad, Hidalgo \#2525 Pte, Col. Obispado, Monterrey N.L. México, CO 64060

Recibido el 15 de mayo de 2014; aceptado el 27 de noviembre de 2014

\section{PALABRAS CLAVE \\ Ablación por catéter; Denervación renal; Hipertensión resistente; Hipertensión arterial; Estudio Symplicity}

\begin{abstract}
Resumen La denervación de las arterias renales ha sido una alternativa para el tratamiento de la hipertensión arterial resistente. Los estudios Symplicity HTN 1 y 2 mostraron en grupos pequeños y no controlados disminuciones de la presión sistólica hasta de $30 \mathrm{~mm} \mathrm{Hg}$. Este entusiasmo ha sido opacado por el estudio Symplicity HTN 3, ensayo clínico aleatorizado y controlado con un procedimiento placebo. Sorprendentemente, los resultados sugirieron que la denervación renal tuvo un efecto similar al del placebo, aunque en el análisis de subgrupos preespecificados los pacientes que no eran de raza negra, los menores de 65 años y los que tenían función renal normal tuvieron una reducción de la presión arterial sistólica estadísticamente significativa. Esta es una evaluación crítica de los resultados del Symplicity HTN 3 y propone posibles explicaciones para estos. Además, declara la postura de nuestro grupo y las acciones futuras.

(C) 2014 Instituto Nacional de Cardiología Ignacio Chávez. Publicado por Masson Doyma México S.A. Todos los derechos reservados.
\end{abstract}

Position paper on the results of Symplicity HTN-3 trial. Grupo de estudio de la hipertensión arterial resistente

\begin{abstract}
Renal artery denervation has shown to be an effective treatment for resistant hypertension. Symplicity HTN 1 and 2 trials showed in small and uncontrolled groups, significant systolic blood pressure reductions down to $30 \mathrm{~mm} \mathrm{Hg}$. Symplicity HTN-3, a double blind, randomized, placebo controlled clinical trial shaded this initial enthusiasm. Surprisingly, their results showed that renal denervation has a similar effect to placebo. Pre-specified subgroup analysis showed that non-black race individuals, younger than 65 years and with normal renal
\end{abstract}

* Autor para correspondencia: Teléfono: +52 818347 8533; fax: +52 8183478534.

Correo electrónico: drazpiri@yahoo.com (J.R. Azpiri-López). 
function, had a statistically significant systolic blood pressure decrease. This manuscript critically appraises the Symplicity HTN-3 trial, proposing possible explanations for the results. Also declares our group position and future actions regarding renal denervation.

(C) 2014 Instituto Nacional de Cardiología Ignacio Chávez. Published by Masson Doyma México S.A. All rights reserved.

El Grupo de estudio de la hipertensión arterial resistente congrega especialistas multidisciplinarios interesados en el manejo de pacientes con hipertensión arterial grave. Se formó a principios de 2013 como órgano regulatorio intrahospitalario, con la finalidad de evaluar los casos de pacientes con hipertensión resistente que fuesen candidatos a denervación renal. Existen reuniones periódicas en donde se revisan los casos, además de información médica relevante relacionada con el tema.

Hace poco se publicó el estudio Symplicity HTN $3^{1}$. Este es un ensayo clínico prospectivo aleatorizado, cegado, controlado con placebo, que compara la respuesta de la presión arterial en 2 grupos de pacientes con hipertensión arterial resistente, y se define como presión arterial sistólica en el consultorio $\geq 160 \mathrm{~mm} \mathrm{Hg}$, tomando al menos 3 medicamentos antihipertensivos a dosis máximas toleradas y que al menos uno de ellos fuera diurético. Los sujetos del estudio se distribuyeron de manera aleatoria para ser tratados con el sistema de denervación renal Symplicity (Medtronic, EUA) o con un procedimiento placebo (angiografía renal selectiva) cuando tenían características anatómicas adecuadas, tales como un solo sistema de arterias renales con diámetro mayor a $4 \mathrm{~mm}$ y longitud superior a $2 \mathrm{~cm}$. La distribución aleatoria fue en relación $2: 1$ con respecto a la cantidad de pacientes que recibieron el procedimiento de investigación comparado con los que recibieron placebo.

El objetivo primario del estudio fue la diferencia entre grupos del cambio promedio de la presión arterial sistólica evaluada en el consultorio del médico a los 6 meses, al compararlo con la previa al tratamiento. El objetivo de seguridad fue la tasa combinada de eventos de muerte por cualquier causa, nefropatía terminal, embolias que causaran daño a algún órgano blanco, complicaciones vasculares renales o estenosis nueva de arteria renal, que de acuerdo con el conocimiento derivado de estudios previos no debería superar el 9,8\%. El objetivo secundario fue la diferencia en la disminución de presión arterial sistólica en el control ambulatorio de la presión arterial. Se evaluaron 1,441 individuos en 88 sitios en Estados Unidos y se excluyeron 2/3 partes de los candidatos. En la mayoría de los centros de estudio no existía experiencia con el dispositivo de investigación ni con el procedimiento. Los pacientes tenían una edad promedio de 57 años; cerca de la mitad eran hombres, con obesidad leve a moderada. Una cuarta parte de la población era de raza negra. El promedio de medicamentos que utilizaban para tratar la hipertensión era de $5.1 \pm 1.4$.

El manuscrito no ofrece detalles técnicos acerca de cómo se realizaron los procedimientos. Los autores no describieron la cantidad y el sitio de las ablaciones, el promedio de caída de la impedancia, la temperatura máxima alcanzada, si se produjeron «indentaciones» angiográficas en las arterias tratadas, si las ablaciones completaron los 2 min o si terminaron el tratamiento en el «techo del orificio», aunque no existe un consenso de que estos se consideren criterios de éxito ${ }^{2-6}$. No pudimos analizar si las ablaciones fueron realizadas de manera apropiada, porque la información para realizar este juicio no está descrita en el manuscrito.

No hubo diferencia entre los grupos en la caída promedio de la presión arterial sistólica medida en el consultorio a los 6 meses. En el grupo de denervación, la caída de presión fue de $14.1 \pm 23.9 \mathrm{~mm} \mathrm{Hg}$ y en el grupo placebo fue de $11.7 \pm 25.9 \mathrm{~mm} \mathrm{Hg}$, para una diferencia de $2.39 \mathrm{~mm} \mathrm{Hg}$ con intervalos de confianza de -6.89 a 2.12; $p=0.26$. Tampoco la hubo en el objetivo secundario de eficacia, el cambio en la presión arterial sistólica en el control ambulatorio de la presión de $24 \mathrm{~h}$, de $6.75 \pm 15.11 \mathrm{~mm} \mathrm{Hg}$ en el grupo de la denervación y de $4.79 \pm 17.25 \mathrm{~mm} \mathrm{Hg}$ en el grupo placebo. En el análisis de subgrupos previamente especificados hubo 3 en los cuales se identificó diferencia estadísticamente significativa. El primero, en los pacientes que no eran de raza negra, hubo una diferencia en la disminución en la presión sistólica medida en el consultorio de $6.63 \mathrm{~mm} \mathrm{Hg}$ (IC 95\% -11.8 a -1.44; p=0.01). El segundo, en pacientes menores de 65 años, con una diferencia en la caída en la presión sistólica de $5.73 \mathrm{~mm} \mathrm{Hg}$ (IC 95\% -11.06 a $-0.40, p=0.04)$. El tercero, en pacientes con tasa de filtración glomerular $\geq 60 \mathrm{ml} / \mathrm{min} / 1.73 \mathrm{~m}^{2}$, con disminución de significación marginal de $5.22 \mathrm{~mm} \mathrm{Hg}$ (IC 95\% - 10.5 a -0.06; $p=0.05)$. No hubo diferencias para el resto de los subgrupos, incluyendo diabetes, género, índice de masa corporal, uso de antagonistas de aldosterona y cambio en el uso de medicamentos. Al aplicar pruebas estadísticas para evaluar las interacciones no se encontraron diferencias significativas entre los subgrupos, así como tampoco las hubo en el objetivo secundario. Estos hallazgos deben considerarse únicamente como generadores de hipótesis solamente ${ }^{7}$.

\section{¿Cuáles son las posibles explicaciones de estos resultados?}

1) Los resultados del estudio sugirieron que la denervación renal tiene el mismo efecto que el placebo. Habrá que explicar cómo ocurrió la disminución cercana a los $11 \mathrm{~mm} \mathrm{Hg}$ en el grupo placebo. Con la información de estudios previos ${ }^{8,9}$, es fácil entender la disminución de la presión en el grupo con tratamiento activo, pero ¿cómo explicar la disminución en el grupo placebo? ¿Padecían 
de verdad hipertensión resistente? ¿Realizaron el escrutinio para descartar hipertensión refractaria? ¿Tomaban correctamente los medicamentos? Es posible que haya existido el fenómeno de regresión a la media, en el cual, después de un tiempo de seguimiento, la mayoría de los parámetros biológicos tienden a normalizarse ${ }^{10}$, aunque esto es poco probable cuando se realiza un estudio aleatorizado. Asimismo, la inclusión en un estudio de investigación puede hacer que los pacientes tengan un mejor control al estar siendo valorados de forma minuciosa, efecto llamado Hawthorne ${ }^{11}$. Otra posibilidad es que los pacientes en el grupo que recibió la denervación se hayan sentido tan bien que de manera voluntaria decidieron modificar las dosis de sus medicamentos, sin notificarlo a los médicos. Se informó de una sensación de bienestar después de la denervación renal ${ }^{12}$. No hay informe de adherencia al tratamiento, ni a modificaciones del estilo de vida, incluyendo la disminución en la ingesta de sodio. La falta de estos datos nos genera dudas acerca de los resultados del estudio. Aun así, es el único estudio controlado con placebo publicado hasta el momento cuyos resultados se aplican a esta población y con este particular sistema de ablación.

2) ¿Realmente se produjo denervación? ¿Es posible que haya ocurrido renervación después de la denervación? Una grave limitación de la manera en que realizamos el procedimiento en la actualidad es que no se determina si en realidad se logró la interrupción de la actividad del sistema nervioso simpático alrededor de las arterias renales. Por eso en el Symplicity HTN 3 no se realizaron maniobras o determinaciones específicas para evaluar el éxito de la denervación, aunque hubiera sido conveniente medir la derrama de norepinefrina o realizar microneurografía clínica, estudios que nos permitirían saber si se logró una denervación efectiva ${ }^{13-15}$. Este tipo de evaluaciones son difíciles de realizar en la práctica diaria. Estos hallazgos pudieran ser la diferencia entre un procedimiento que no es efectivo y uno que simplemente no se realizó. Los próximos estudios deberán incluir la medición de la actividad simpática como evidencia de que el procedimiento se realizó de manera adecuada.

Desde el punto de vista técnico, con el catéter Symplicity deben hacerse flexiones, retracciones y torsiones entre cada sitio que recibirá la ablación, lo cual aumenta la complejidad e incertidumbre del procedimiento. Es posible que otros dispositivos que logren un mejor adosamiento a la pared de la arteria generen ablaciones más homogéneas y puedan tener resultados diferentes. Por ello, no deben extrapolarse los resultados del estudio Simplicity HTN-3 comparándolo con otros dispositivos que actualmente continúan en estudio.

Se ha demostrado en modelos de trasplante renal que los nervios simpáticos tienen la capacidad de regenerarse. En modelos porcinos existe un intento de regeneración nerviosa tan temprana como 7 días después de la terapia de denervación renal, evaluada por inmunohistoquímica, aunque debe demostrarse que esta sea efectiva mediante estudios funcionales ${ }^{16}$. La renervación puede explicar en teoría la falta de efectividad del procedimiento, al menos en algunos pacientes.

3) Error tipo beta; el tamaño de la muestra. En el manuscrito se comenta que el cálculo del tamaño de la muestra se realizó usando los puntos terminales de seguridad y eficacia, y se compara la tasa de eventos adversos con un parámetro de «desempeño óptimo» asumiendo una tasa de morbilidad de procedimientos intervencionistas en las arterias renales de $9.8 \%$. Entonces, si se tuviese una tasa de eventos adversos inferior a 7.0\%, con 316 pacientes en el grupo de tratamiento el estudio tendría un poder de 0.8 a un nivel alfa de 0.05 . Debido a que la distribución aleatoria fue 2:1, requirieron 158 pacientes en el grupo control. En el manuscrito no se especifica cómo hicieron el cálculo con base en el punto terminal de efectividad, y se refiere al lector a otro trabajo en el cual se describió el protocolo ${ }^{17}$. En este se explica el cálculo del poder del estudio más que el cálculo del tamaño de la muestra. Se asume que con el tamaño de muestra estimado $(n=316)$ el estudio tendría un poder de 0.9 a un nivel alfa de 0.025 para detectar una diferencia de $15 \mathrm{~mm} \mathrm{Hg}$ entre los 2 grupos, con una desviación estándar de $25 \mathrm{~mm} \mathrm{Hg} \mathrm{en}$ cada grupo. En el reporte de los resultados se comunica que una reducción apropiada sería $5 \mathrm{~mm} \mathrm{Hg}$ de diferencia entre los grupos y no explican el cálculo del tamaño de la muestra para detectar esta diferencia.

Asumiendo una diferencia en la presión arterial entre grupos de 5 puntos, con una desviación estándar de 26 puntos en un grupo y 24 en el otro, se requieren cerca de 400 pacientes por grupo para demostrar una diferencia estadísticamente significativa a un nivel alfa de 0,05 con un poder de 0,8. El tamaño de la muestra en el estudio es inferior al que hubiera sido necesario para probar efectividad y posiblemente se incurra en error tipo beta (cálculo realizado con el paquete estadístico Med Calc, Ostend, Bélgica).

4) ¿Diferencias individuales? El $25 \%$ de los pacientes de raza negra que participaron en el estudio determinaron que este fuera negativo. Si no se hubiesen incluido, el estudio mostraría superioridad a nivel alfa de 0.01 , como lo sugiere el análisis de subgrupos. Este puede ser el factor clave del porqué los estudios de los alemanes y los australianos tienen resultados tan favorables, aunque carecen de control con placebo ${ }^{2,8,18-20}$. Es imperativo realizar un estudio prospectivo, aleatorizado y controlado con placebo que excluya pacientes con estas características raciales, ya que no podremos saber si la falta de efecto a la denervación es por ineficaz o por diferencias raciales en la respuesta al procedimiento. Será también importante identificar otras metodologías que pudiesen predecir la respuesta al tratamiento. Dorr et al. reportaron que sujetos sometidos a denervación renal que tenían marcadores de disfunción endotelial elevados como sFLT-1, ICAM-1 y VCAM-1 tenían mejor respuesta al tratamiento que aquellos con cifras normales, y esto ofrece una posibilidad para explicar las diferentes respuestas en el tratamiento y posiblemente predecir los resultados $^{21}$.

\section{Conclusiones}

1) El primer estudio aleatorizado controlado con un procedimiento placebo («falso»), comparando la denervación renal con el catéter Symplicity de Medtronic y el tratamiento médico óptimo en pacientes con hipertensión 
arterial resistente, sugiere que la denervación renal con este catéter en particular no demostró diferencias entre ambos grupos. Con sus limitaciones, esta es la evidencia científica más sólida relacionada con el procedimiento y su posible beneficio hasta el momento.

2) En el estudio Symplicity HTN 3 hubo 3 subgrupos que se beneficiaron: los sujetos que no eran de raza negra, los menores de 65 años y los individuos con función renal normal. Estos hallazgos son solamente generadores de nuevas hipótesis.

3) Consideramos pertinente continuar realizando denervaciones renales en sujetos con hipertensión arterial sistémica primaria refractaria a 3 medicamentos en dosis máximas toleradas que incluya un diurético, con función renal normal, con características anatómicas apropiadas previa presentación al comité correspondiente. Todos los pacientes estarán informados correctamente acerca de los riesgos y posibles beneficios del procedimiento, siempre apegados a los principios éticos y a la normatividad, misma que detonó el origen del presente comité. Se les seguirá informando de que el papel definitivo de la denervación renal para el tratamiento de la hipertensión arterial resistente continúa en investigación y que sus datos demográficos, del procedimiento y del seguimiento seguirán recabándose de manera protocolizada.

\section{Financiación}

No se recibió patrocinio de ningún tipo para llevar a cabo este artículo.

\section{Conflicto de intereses}

Los autores declaran no tener ningún conflicto de intereses.

\section{Bibliografía}

1. Bhatt DL, Kandzari DE, O’Neill WW, et al. A controlled trial of renal denervation for resistant hypertension. N Engl J Med. 2014;370:1393-401.

2. Krum H, Schlaich M, Whitbourn R, et al. Catheter-based renal sympathetic denervation for resistant hypertension: A multicentre safety and proof-of-principle cohort study. Lancet. 2009;373:1275-81.

3. Mahfoud F, Luscher TF, Andersson B, et al. Expert consensus document from the European Society of Cardiology on catheterbased renal denervation. Eur Heart J. 2013;34:2149-57.

4. Bertog SC, Blessing E, Vaskelyte L, et al. Renal denervation: Tips and tricks to perform a technically successful procedure. Eurointervention. 2013;9 Suppl R:R83-8.

5. Gaspar J. Fundamentos y evidencias para la ablación por radiofrecuencia de la arteria renal en la hipertensión arterial resistente. Avances Cardiol. 2013;33:40-4.
6. Scheurig-Muenkler C, Weiss W, Foert E, et al. Renal denervation for refractory hypertension-technical aspects, complications and radiation exposure. Fortschr Röntgenstr. 2013;185: 550-7.

7. Wang R, Lagakos SW, Ware JH, et al. Statistics in medicinereporting of subgroup analyses in clinical trials. N Engl J Med. 2007;357:2189-94.

8. Esler MD, Krum H, Sobotka PA, et al. Renal sympathetic denervation in patients with treatment-resistant hypertension (The Symplicity HTN-2 Trial): A randomised controlled trial. Lancet. 2010;376:1903-9.

9. Esler MD, Krum H, Schlaich M, et al. Renal sympathetic denervation for treatment of drug-resistant hypertension: One-year results from the Symplicity HTN-2 randomized, controlled trial. Circulation. 2012;126:2976-82.

10. Dawson B. Preguntas en investigación sobre relaciones entre variables. En: Trapp BDRG, editor. Bioestadística médica. 3a ed. México: El Manual Moderno; 2002. Pág. 224.

11. McCambridge J, Witton J, Elbourne DR. Systematic review of the Hawthorne effect: New concepts are needed to study research participation effects. Journal Clin Epidemiol. 2014;67: 267-77.

12. Lambert GW, Hering D, Esler MD, et al. Health-related quality of life after renal denervation in patients with treatment-resistant hypertension. Hypertension. 2012;60:1479-84.

13. Esler M, Jennings $G$, Korner $P$, et al. Assessment of human sympathetic nervous system activity from measurements of norepinephrine turnover. Hypertension. 1988;11:3-20.

14. Lambert EA, Schlaich MP, Dawood T, et al. Single-unit muscle sympathetic nervous activity and its relation to cardiac noradrenaline spillover. J Physiol. 2011;589:2597-605.

15. Burke SL, Lambert E, Head GA. New approaches to quantifying sympathetic nerve activity. Curr Hypertens Rep. 2011;13: 249-57.

16. Garza JAHR, Wicks J. Evaluation of the acute and long term renal artery re-innervation attempt response following radiofrequency catheter-based renal nerve ablation in a swine model: An immunohistochemical characterization. Rev Mex Cardiol. 2014;25:S3.

17. Kandzari DE, Bhatt DL, Sobotka PA, et al. Catheter-based renal denervation for resistant hypertension: rationale and design of the Symplicity HTN-3 Trial. Clin Cardiol. 2012;35:528-35.

18. Kaltenbach B, Franke J, Bertog SC, et al. Renal sympathetic denervation as second-line therapy in mild resistant hypertension: A pilot study. Catheter Cardiovasc Interv. 2013;81: 335-9.

19. Ott C, Mahfoud F, Schmid A, et al. Renal denervation in moderate treatment-resistant hypertension. J Am Coll Cardiol. 2013;62:1880-6.

20. Kaiser L, Beister T, Wiese A, et al. Results of the ALSTER BP realworld registry on renal denervation employing the Symplicity system. Eurointervention. 2014;10:157-65.

21. Dorr O, Liebetrau C, Mollmann H, et al. Soluble fms-like tyrosine kinase-1 and endothelial adhesion molecules (intercellular cell adhesion molecule-1 and vascular cell adhesion molecule-1) as predictive markers for blood pressure reduction after renal sympathetic denervation. Hypertension. 2014;63: 984-90. 